\title{
Conexinas y exosomas: dos mecanismos de comunicación entre las células tumorales
}

\section{Connexins and exosomes: two mechanisms of communication in tumor}

Laura G. Hernández-Aragón', José Everardo Avelino-Cruz², Carlos Alberto Pacheco-Pérez³, Fabián Galindo-Ramírez ${ }^{4, *}$

1. Doctora en Ciencias Biológicas, Laboratorio de Cáncer y Comunicación Intercelular, Instituto de Fisiología, Benemérita Universidad Autónoma de Puebla.

2. Doctor en Fisiología y Neurociencias, Laboratorio de Cardiología molecular, Instituto de Fisiología, Benemérita Universidad Autónoma de Puebla.

3. Estudiante de biotecnología, Laboratorio de Cáncer y Comunicación Intercelular, Instituto de Fisiología, Benemérita Universidad Autónoma de Puebla.

4. Doctor en Ciencias Fisiológicas, Jefe del Laboratorio de Cáncer y Comunicación Intercelular, Instituto de Fisiología, Benemérita Universidad Autónoma de Puebla.

*Autor de correspondencia: Fabián Galindo-Ramírez

Dirección: 14 Sur, 6301, Colonia San Manuel, Ciudad Universitaria C.P. 72570. Puebla. Puebla, México.

Email: fabgalindoram@gmail.com

DOI http://dx.doi.org/10.28960/revmeduas.2007-8013.v12.n1.009

Recibido 27 de julio 2021, aceptado 04 de octubre 2021

\section{RESUMEN}

Entender los mecanismos de comunicación que emplean las células tumorales podría ayudarnos mejorar las terapias para combatir diferentes tipos de cáncer. Las conexinas y los exosomas forman parte de estos mecanismos, las primeras forman canales en la membrana celular y las segundas son vesículas extraordinariamente pequeñas. Ambas tienen un enorme potencial para el tratamiento y erradicación del cáncer.

Palabras Clave: Cáncer, conexinas, exosomas.

\section{ABSTRACT}

Understand mechanism of communication that tumoral cells employed could help improve therapies to combat different types of cancer. Connexins and exosomes are part of this mechanism, the first ones conform channels in cellular membrane and second ones are a vesicle extremely small. Both have enormous potential for cancer treatment and eradication.

Keywords: Cancer, Connexins, exosomes.

\section{Introducción}

Las células, requieren maneras de comunicarse tanto en su interior para optimizar funciones, como al exterior, ya sea para conocer el microambiente que las rodea, compartir información con otras células cercanas o bien, enviar señales a sitios lejanos. Al agruparse y formar tejidos deben funcionar de manera armónica y coordinada para mantener las funciones para las cuales están diseñadas, por ello la comunicación intercelular es fundamental. En términos generales, se ha descrito que lo anterior sucede por la emisión de un mensaje, generalmente una señal química, que al llegar a su destino (otra célula), ejerce su efecto, ocasionando una respuesta biológica, la cual, es responsable de generar cambios morfológicos, modificación de 
rutas metabólicas o incluso, cambios en la expresión de genes, llevando así a procesos de división, diferenciación, sobrevivencia o muerte celular ${ }^{1}$. Por esta razón, la comunicación celular juega un papel fundamental en el mantenimiento de la homeostasis en condiciones de salud, así como en el desarrollo de las enfermedades. El cáncer, es una patología en la cual se ha desarrollado una increíble capacidad para comunicar mensajes erróneos, mediante la desregularización y disrupción de diversas señales que favorecen el crecimiento y propagación de células tumorales pero que en condiciones normales son esenciales para el mantenimiento de las funciones fisiológicas ${ }^{2}$.

Actualmente, el cáncer es una de las diez primeras causas de muerte a nivel mundial, según la Organización Mundial de la Salud (OMS), 2020 y se registraron 9.9 millones de defunciones para el término del $2020^{3}$. Se estima que para el 2040, el número de nuevos casos aumentará de 19.3 millones a aproximadamente 30.2 millones, representando un incremento del 156 \%. En México, se presentaron 195499 nuevos casos para el 2020 y se registraron un total de 90222 defunciones para este mismo año, se espera un incremento de 323000 nuevos casos para el año 2040 , lo que representa un $165 \%{ }^{4}$. Según la OMS (2021), el cáncer es una enfermedad caracterizada por el aumento incontrolado del número de células de un tejido específico, este aumento lleva a la generación de un tumor el cual, puede causar diferencies síntomas, dependiendo de su localización y en caso de no ser tratado, su tamaño aumentará hasta interferir con sistemas vitales causando la muerte del organismo ${ }^{4}$. El cáncer es una enfermedad multifactorial, es decir, se origina por la suma de muchas alteraciones de los procesos vitales de las células. En condiciones no patológicas, las células que acumulan cambios inesperados (mutaciones) en su código genético (ADN) son desechadas, sin embargo, estas mismas mutaciones pueden llegar a dañar el sistema encargado de efectuar su detección y eliminación ${ }^{5}$. Cuando esto sucede la célula se sigue reproduciendo hasta formar una masa de células llamada tumor.

\section{El microambiente tumoral.}

El microambiente tumoral es un entorno dinámico y privilegiado, su composición bioquímica y celular es de primordial importancia para la regulación del metabolismo, proliferación, motilidad y diseminación de las células tumorales ${ }^{6}$. Las células cancerígenas se pueden comunicar con aquellas que se encuentran a su alrededor. Estas envían señales que les permiten crecer y transformar a otras células sanas en células tumorales ${ }^{7}$. Además, utilizan estas señales para comunicar sus necesidades al tejido circundante. Una característica importante en el microambiente tumoral es la hipoxia, es decir, la disminución en la concentración de oxígeno. 
Esto se debe a que al estar en constante crecimiento las células cancerígenas necesitan un suministro de nutrientes mayor, entre ellos el oxígeno. Para contrarrestar la deficiencia de oxígeno y mantenerse en constante crecimiento, generan nuevos vasos sanguíneos, los cual les proveen sangre repleta de nutrientes, a este proceso se le llama angiogénesis ${ }^{8}$.

Las células nunca están solas, siempre están rodeadas de más células y moléculas con las cuales interactúan constantemente. Particularmente, se ha observado que las células tumorales interactúan como su microambiente, lo cual contribuyen a la progresión del tumor y a la propagación de células tumorales a otros sitios, un proceso conocido como metástasis ${ }^{9}$. El microambiente tumoral también se compone de productos generados por células del estroma, estas son células que rodean al tumor como: los fibroblastos, células del sistema vascular, y células del sistema inmune, así como de factores solubles, tales como los factores de crecimiento, hormonas y citocinas ${ }^{6,10}$. Dado que este microambiente afecta la interacción célula-célula, la comunidad científica ha reconocido que la mejora en las terapias contra el cáncer deben considerar el microambiente tumoral como un posible blanco terapéutico o complementario a los tratamientos dirigidos a las células cancerígenas ${ }^{11}$.

\section{Comunicación intercelular.}

De acuerdo al modo en que se trasporta la señal hacia otra célula, se han clasificado en 4 mecanismos principales de comunicación. El primero que se mencionará es el endócrino, caracterizado porque la señal recorre una mayor distancia y utilizando como mediador hormonas que son secretadas al sistema circulatorio. El segundo es el parácrino, donde los mediadores son secretados al ambiente extracelular y actúan de forma local, en células del tejido cercano. El tercero es el yuxtácrino, en el cual actúa en las células se encuentren adyacentes. Por último, tenemos al autócrino, en donde las moléculas son liberadas al espacio extracelular, pero actúan sobre la misma célula que las liberó $^{12}$, (figura 1).

Figura 1. Principales tipos de comunicación celular. Esquema representativo de la comunicación autócrina, yuxtácrina, paracrína y endocrína.

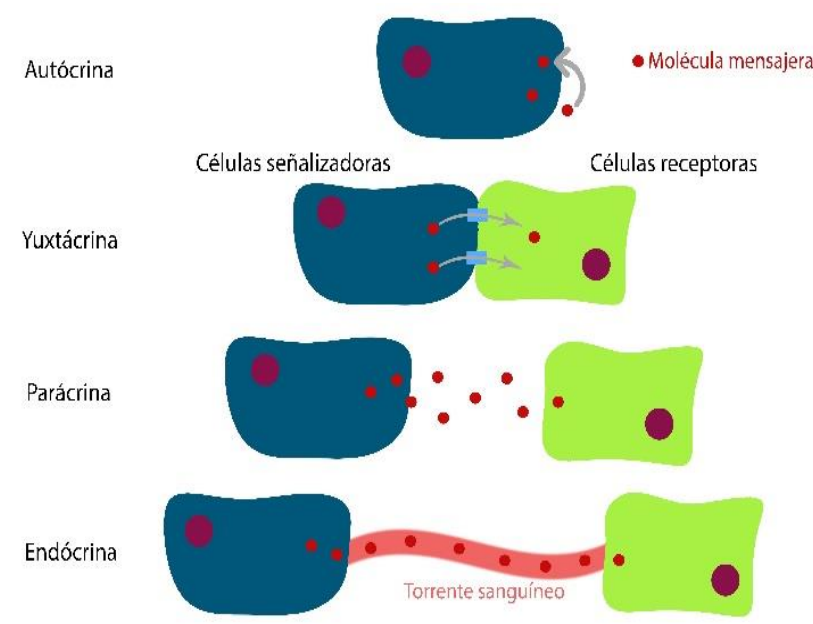


Las señales químicas liberadas al espacio extracelular que se generan en una célula pueden ser enviadas a células adyacentes, y algunas pueden difundir a través de la membrana celular, dado que es semipermeable. Pero, también pueden incorporarse mediante canales, para interaccionar con elementos del citoplasma o del propio núcleo, o bien, se unen a receptores en la membrana que activan una serie de reacciones al interior de la célula. Por otra parte, la comunicación yuxtácrina está dada por canales que pueden formar un puente directo entre dos células, conectando directamente sus citoplasmas. Estos canales pueden estar conformados por diferentes tipos de proteínas, en particular aquellos que son de interés para esta revisión, son las conexinas, las cuales pueden formar un tipo de unión conocida como comunicante o en hendidura (gap junctions, en inglés) ${ }^{13}$.

\section{Uniones comunicantes}

Las conexinas son proteínas constituidas por 4 dominios transmembrana, es decir, 4 segmentos que atraviesan la membrana plasmática en su totalidad y están unidos por 2 lazos en la parte exterior y uno al interior de la célula (figura 2a). Al unir 6 conexinas se forma un conexón o hemicanal, el cual es un canal funcional ${ }^{14}$ (figura $2 b)$. Dependiendo el tipo de conexina y el tejido donde se encuentre el conexón o hemicanal será la función que realice. Dentro de las moléculas que pueden atravesar la membrana por medio de estos se encuentran: Adenosín trifosfato (ATP), empleado para el almacenamiento de energía y como mensajero químico; $\mathrm{NAD+}$, utilizado para el transporte de electrones; glutamato, el cual actúa como neurotransmisor; iones como $\mathrm{Ca}^{2+}$ y $\mathrm{Na}^{+}$, entre otros ${ }^{15}$ (figura 2c). Cuando dos células vecinas se están comunicando, el conexón de una puede interaccionar con el de la otra para formar la unión comunicante o en hendidura (figura $2 \mathrm{~d}$ ).

En células humanas se han detectado 21 diferentes tipos de conexinas y 20 en ratones, estas reciben su nombre con base a su tamaño predicho en kiloDaltons $(\mathrm{kDa})$, donde un Dalton es definido como una doceava parte de la masa de un átomo de carbono-12. En un mismo tipo celular pueden estar presentes diferentes tipos de conexinas, cada una de ellas con diferente forma, tamaño y función. Por ejemplo, en células neuronales de ratón están presentes $\mathrm{Cx} 36$ y Cx45, en cambio, se han detectado Cx26 y Cx32 en células hepáticas ${ }^{16}$. 
Figura 2. Estructura y función de una conexina, conexón/hemicanal y unión comunicante. a) Cada conexina está formada por 4 dominios transmembrana unidos por dos lazos extracelulares y uno intracelular. b) Los cuatro dominios se pliegan para formar la conexina. Un conexón/hemicanal se forma cuando 6 conexinas se unen, pueden estar en conformación cerrada, impidiendo el paso de moléculas a través de ellos, o abierta. c) Por un conexón/hemicanal en conformación abierta pueden pasar distintas moléculas tanto hacia el interior como al exterior. d) Al estar dos células próximas dos conexones pueden unirse permitiendo el intercambio de moléculas entre sus citoplasmas.

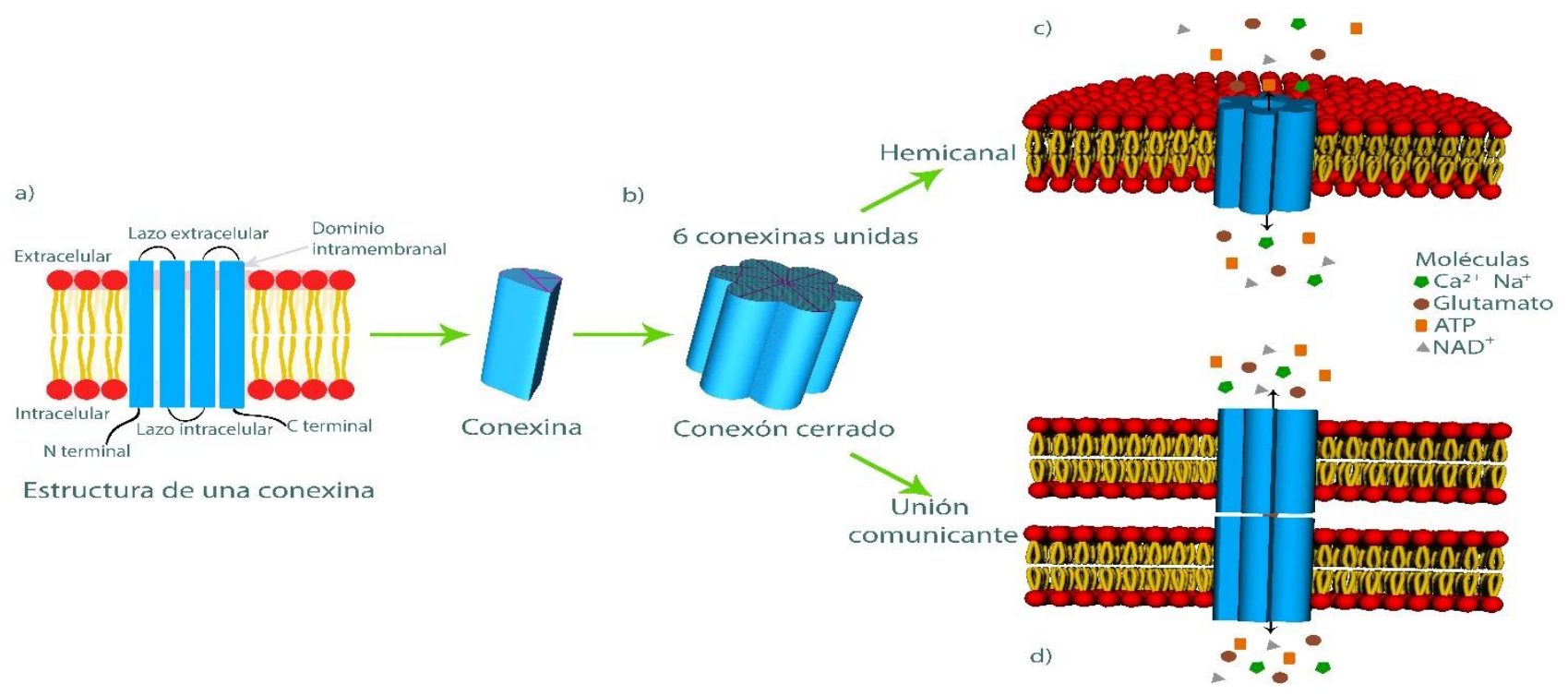

\section{Los exosomas}

La comunicación paracrina también participa de manera fundamental en el desarrollo de un tumor, una manera es mediante la liberación de vesículas que viajan hasta la célula objetivo afectando su actividad y función. Ejemplo de ello son los exosomas, los cuales son un subconjunto de vesículas extracelulares que tienen una forma de "plato" cuando se observan en el microscopio electrónico. Contienen moléculas de la célula de origen, incluyendo proteínas, lípidos, y ácido ribonucleico (ARN) de diferentes tipos (figura 3). A la fecha, al interior de los exosomas se han identificado miles de proteínas, incluso material genético provenientes de distintos tipos celulares.
La primera descripción de los exosomas fue hecha en 1980 por Rose Johnstone donde proponía que solo eran liberados por los glóbulos rojos inmaduros ${ }^{17}$, sin embargo, los exosomas son secretados por varios tipos celulares: tanto células hematopoyéticas (linfocitos $\mathrm{T}$ y $\mathrm{B}$, plaquetas, células dendríticas y macrófagos), así como, por células de origen no hematopoyético (células de Schwann, astrocitos, fibroblastos y células tumorales $)^{18}$. Se ha demostrado que las células tumorales son capaces de liberar una mayor cantidad de exosomas al medio extracelular que las células normales ${ }^{19}$. Estos son liberados a fluidos biológicos como: orina, líquido amniótico, saliva, linfa, líquido cefalorraquídeo y sangre, por medio de los cuales son transporta- 
dos a regiones distantes de su célula de origen $^{20}$. Al principio se creía que servían de "bolsas de basura", es decir, para la eliminación de material no deseado por las células, sin embargo, su función es aún más compleja, pues estas vesículas juegan un papel esencial en la comunicación intercelular. Recientemente se les ha implicado en diversas enfermedades entre ellas el cáncer, pues participan activamente en la transformación de células sanas a tumorales, resistencia a fármacos, y transporte de factores tumorales ${ }^{21}$. Por lo tanto, las células tumorales parecieran utilizar a los exosomas como mensajeros para transformar a las células vecinas y crear un ambiente ideal para su crecimiento y expansión ${ }^{22}$. De tal manera que la estructura y función de los exosomas pueden hacerlos útiles en el diagnóstico y pronóstico del cáncer, de esta forma, podrían ser empleados para una temprana detección o control de esta enfermedad ${ }^{20,23}$.

La forma en cómo se generan los exosomas no está totalmente definida, sin embargo, modelos actuales sugieren que se liberan mediante la fusión de cuerpos multivesiculares (MVBs) con la membrana plasmática (Figura 3).

\section{Figura 3: Biogénesis de exosomas mediante cuerpos multivesiculares}

Las vesículas plasmáticas pueden contener exosomas provenientes directamente de la membrana plasmática o por la fusión de compartimentos multivesiculares (MVB) con la membrana plasmática. Los exosomas contenidos en una vesícula pueden ser una población heterogénea y son liberados por la célula cuando el exosoma tardío se funciona con la membrana plasmática o bien, son degradados mediante lisosomas. Imagen modificada Colombo24.

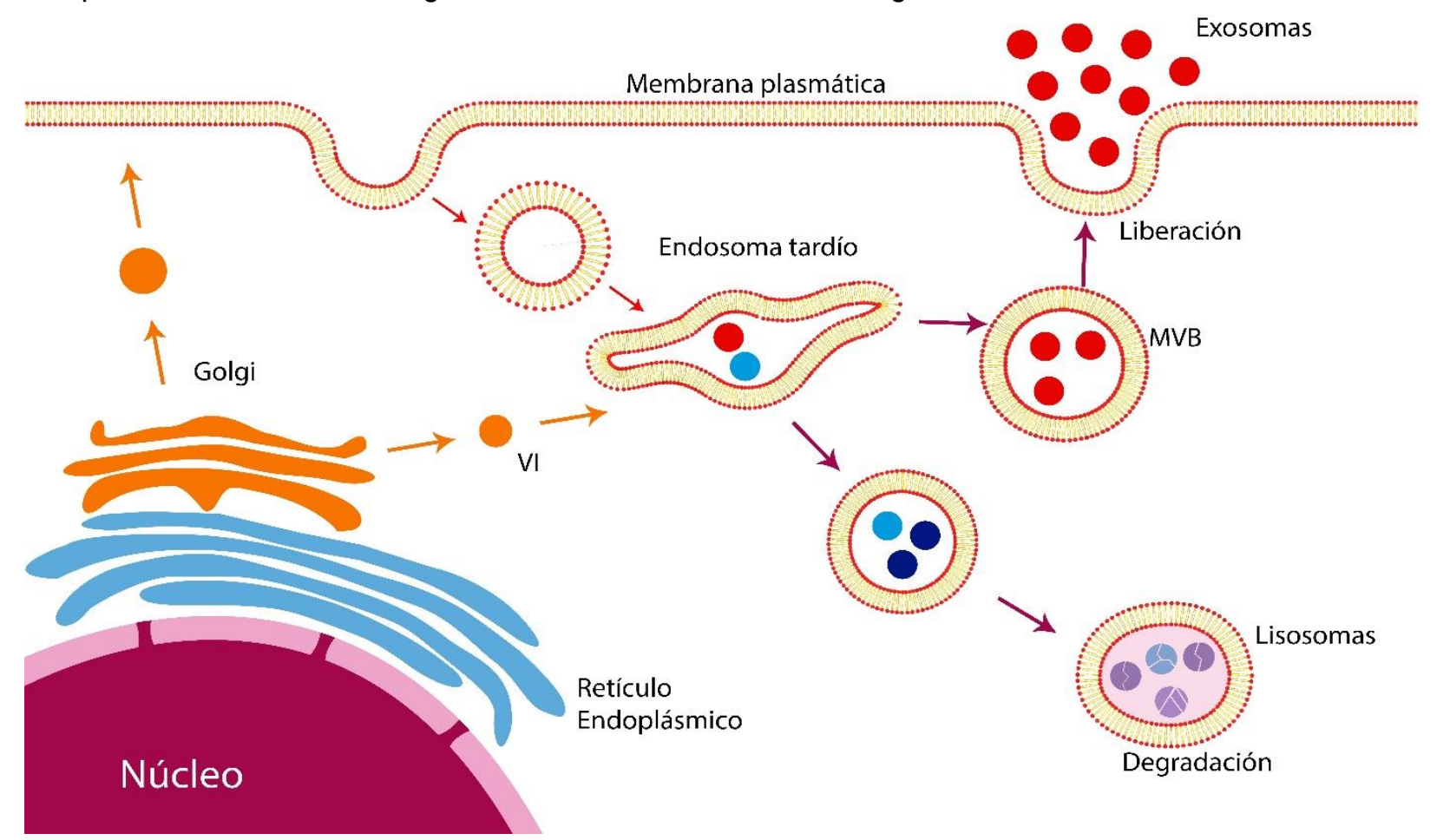


La secreción de exosomas puede verse afectada por varios mecanismos, entre ellos; aumento intracelular de calcio, estrés y disminución de oxígeno ${ }^{17}$.

El flujo de información que se obtiene tanto de las uniones comunicantes como de la liberación de exosomas no es constante, cambia en función de la edad, del estado fisiológico, de estímulos del medio ambiente, así como la salud del organismo. Un cambio anormal en estos mecanismos puede causar o puede ser causado por alguna enfermedad, alterando la cantidad o el tipo de moléculas secretadas y agravando el padecimiento.

\section{Comunicación intercelular y cáncer}

Las células tumorales tienen la capacidad de migrar, para poder hacerlo deben de superar diversos obstáculos como lo son la matriz extracelular, la pared de los vasos sanguíneos o linfáticos y sobrevivir a las células de defensa. La conexina 43 (Cx43) se encuentra ampliamente distribuida en células del sistema ${ }^{25}$. Se ha demostrado que en cáncer de cerebro o de médula espinal (glioma), la cantidad de conexinas se ve alterada, siguiendo una correlación negativa entre la síntesis de Cx43 y capacidad de invasión de las células, es decir, a menor cantidad de dicha conexina existe una mayor probabilidad de realizar metástasis ${ }^{26}$. En el caso del glioblastoma, el tipo de glioma con peor pronóstico, también es aquel que expresa la menor cantidad del conexón formado por Cx43. Sin embargo, se ha demostrado que no es necesario la ausencia del conexón completo para aumentar la probabilidad de realizar metástasis ${ }^{27}$. Esto se descubrió en células de ratón a las cuales se les eliminó el carboxilo terminal del conexón y se observó el incremento en la movilidad celular (metástasis), lo cual, fue confirmado en células sin Cx43 introduciendo solamente el carboxilo terminal y observando una reducción en la metástasis y en la división celular ${ }^{28}$.

En nuestro laboratorio, trabajando con líneas celulares tumorales, estamos estudiando la participación de las uniones comunicantes y conexones en la propagación de señales que ayuden a disminuir el número de células malignas mediante la generación de procesos de muerte. En particular, la Cx43 se encuentra ampliamente expresada en líneas tumorales de mama, próstata y pulmón, tanto de origen humano como murino ${ }^{29}$. La presencia de conexinas (Cx43 y Cx46) favorecen la propagación de señales que ocasionan la muerte de las células que rodean a aquella que ha sido estimulada mediante un fotoestímulo. Estudios in vitro en células de adenocarcinoma de ratón han demostrado que el bloqueo de uniones comunicantes, particularmente dado por conexinas, disminuye la propagación de señales de muerte celular. Las moléculas que están participando de manera activa en este proceso son el $\mathrm{Ca}^{2+} \mathrm{y}$ las especies reactivas de oxígeno ${ }^{30}$. 
En relación a los exosomas, se sabe que las células cancerígenas son capaces de liberarlos y estos llevan en su interior oncogenes y otras moléculas relacionadas con el desarrollo tumoral, las cuales no solo modifican a las células vecinas, sino que pueden viajar a través de los fluidos corporales y llegar a células blanco lejanas en diferentes tejidos ${ }^{22}$. El papel de los exosomas en el cáncer es multifactorial ya que están relacionados con la iniciación, crecimiento, progresión, resistencia a fármacos, inmunosupresión, interacciones con el medio ambiente tumoral, formación de nuevos vasos sanguíneos (angiogénesis), ayudando a la colonización de nuevos órganos (metástasis) y diferenciación de fibroblastos favoreciendo la remodelación del espacio que rodea al tumor incrementando su probabilidad de crecer ${ }^{31}$. Se ha demostrado que los exosomas están implicados en la inducción de muerte de las células del sistema inmune responsables de atacar al tumor, lo que genera un estado conocido como inmunosupresión ${ }^{22}$ (figura 4).

Figura 4: Posibles acciones debidas a la liberación exosomal de células cancerígenas. Acciones locales y sistémicas de los exosomas que favorecen la progresión de células tumorales, así como los procesos metastásicos.

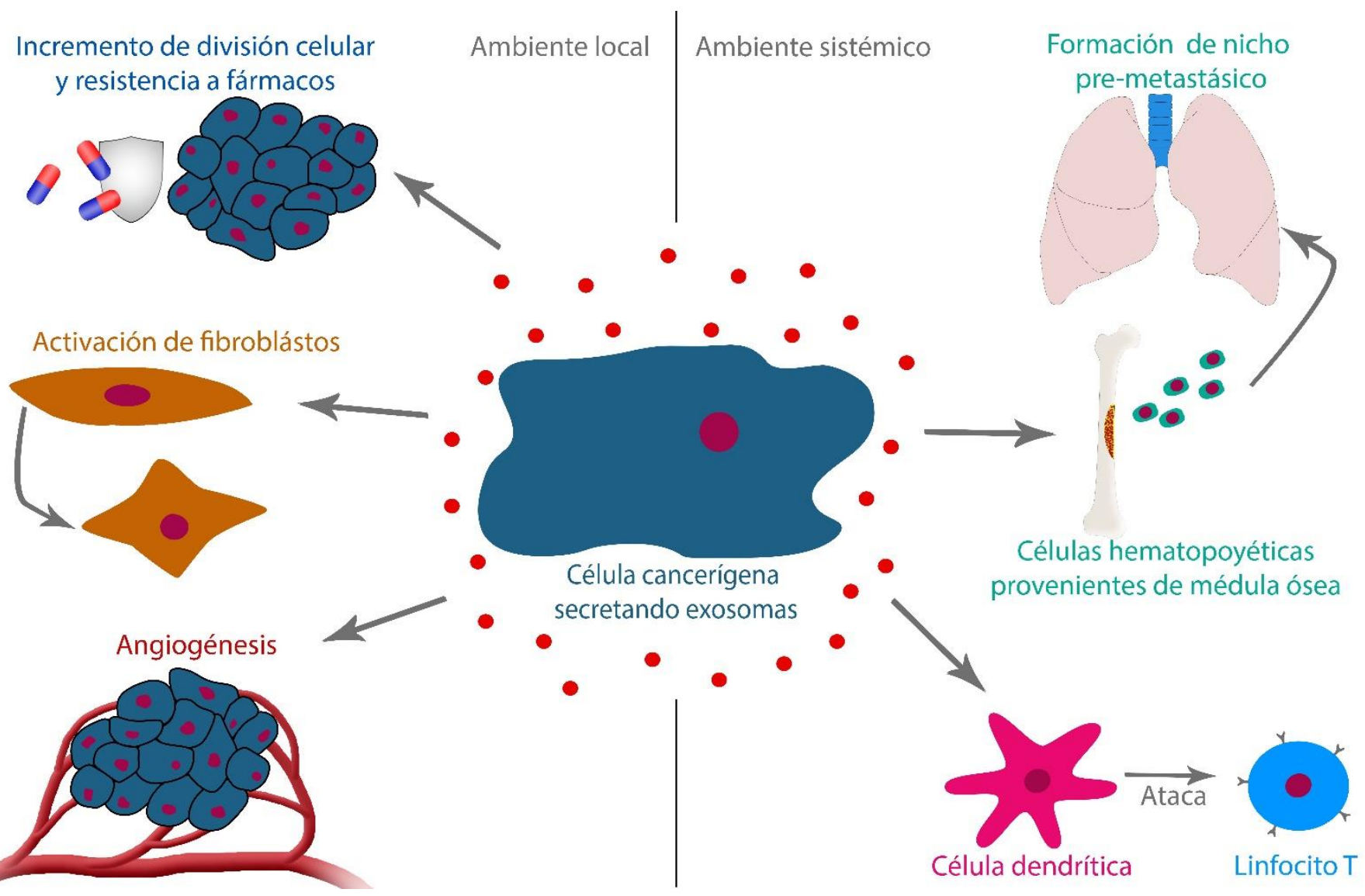


Estudios en líneas celulares de cáncer de mama, han confirmado que los exosomas llevan consigo moléculas con antígenos expresados en la superficie celular. De hecho, las vesículas provenientes de células de cáncer de mama y de glioblastoma demostraron ser capaces de conferir características tumorales a las células de mama normales y a fibroblastos ${ }^{31}$. Entre estas propiedades se encuentran el incremento de su supervivencia, así como la habilidad para vivir en condiciones pobres de nutrientes y de factores de crecimiento externos. Esta es una evidencia directa de que las células normales adquieren características malignas cuando son expuestas a exosomas provenientes de células tumorales, por lo tanto, el incremento de masa tumoral no solo depende de la división celular sino también de los exosomas, los cuales están provocando la transformación de las células en la periferia del tumor ${ }^{32}$. Por otra parte, se ha demostrado que los exosomas provenientes de las células de cáncer de vejiga, promueven la proliferación e inhiben la apoptosis, es decir, la muerte programada de las células $^{33}$.

\section{Consideraciones finales}

La comunicación intercelular juega un papel protagónico en la desarrollo y proliferación del cáncer, en particular, aquella mediada por conexinas, mediante la formación de conexones o uniones comunicantes y la que depende de los exosomas, lo anterior mostrado, nos brindan una idea de la enorme complejidad que existe en la fisiopatología de esta enfermedad. Actualmente, el cáncer es una de las enfermedades que ocasiona mayor número de muertes a nivel mundial, por lo que entender la forma en la que esta comunicación se regula y los mensajes que pueden ser enviados a través de ella, nos podría brindar la oportunidad de generar nuevos tratamientos, con mayor especificidad, eficacia y en especial, con el menor número de efectos secundarios.

\section{Referencias}

1. Stahl PD, Raposo G. Extracellular Vesicles: Exosomes and Microvesicles, Integrators of Homeostasis. Physiology (Bethesda). 2019;34(3):169-177.

2. AlMusawi S, Ahmed M, Nateri AS. Understanding cell-cell communication and signaling in the colorectal cancer microenvironment. Clin Transl Med. 2021;11(2): e308.

3. GLOBOCAN (2021) Cancer today, disponible en http://gco.iarc.fr/).

4. Instituto Nacional de Cáncer, NIH (2021), disponible en https://www.cancer.gov/espanol/cancer/naturaleza/que-es

5. Brown AL, Li M, Goncearenco A, Panchenko AR. Finding driver mutations in cancer: Elucidating the role of background mutational processes. PLoS Comput Biol. 2019;15(4):e1006981.

6. Arneth B. Tumor Microenvironment. Medicina (Kaunas). 2019;56(1):15. 
7. Cooper GM. The Cell: A Molecular Approach. 2nd edition. Sunderland (MA): Sinauer Associates; 2000. The Development and Causes of Cancer. Available: https://www.ncbi.nlm.nih.gov/books/NBK99 63/

8. Jing X, Yang F, Shao C, Wei K, Xie M, Shen $\mathrm{H}$, Shu Y. Role of hypoxia in cancer therapy by regulating the tumor microenvironment. Mol Cancer. 2019;11,18(1):15

9. Hinshaw DC, Shevde LA. The Tumor Microenvironment Innately Modulates Cancer Progression. Cancer Res. 2019;79(18):4557-4566.

10. Denton AE, Roberts EW, Fearon DT. Stromal Cells in the Tumor Microenvironment. Adv Exp Med Biol. 2018; 1060:99-114.

11. Hui L, Chen Y. Tumor microenvironment: Sanctuary of the devil. Cancer Lett. 2015;368(1):7-13.

12. Brücher BL, Jamall IS. Cell-cell communication in the tumor microenvironment, carcinogenesis, and anticancer treatment. Cell Physiol Biochem. 2014;34(2):213-243.

13. Beyer EC, Berthoud VM. Gap junction gene and protein families: Connexins, innexins, and pannexins. Biochim Biophys Acta Biomembr. 2018;1860(1):5-8.

14. Nielsen MS, Axelsen LN, Sorgen PL, Verma V, Delmar M, Holstein-Rathlou NH. Gap junctions. Compr Physiol. 2012;2(3):19812035.

15. Harris AL. Connexin channel permeability to cytoplasmic molecules. Prog Biophys Mol Biol. 2007;94(1-2):120-143.
16. Kar R, Batra N, Riquelme MA, Jiang JX. Biological role of connexin intercellular channels and hemichannels. Arch Biochem Biophys. 2012;524(1):2-15.

17. Johnstone RM. Exosomes biological significance: A concise review. Blood Cells Mol Dis. 2006;36(2):315-321.

18. Zhang J, Li S, Li L, Li M, Guo C, Yao J, Mi S. Exosome and exosomal microRNA: trafficking, sorting, and function. Genomics Proteomics Bioinformatics. 2015;13(1):17-24

19. Zhang L, Yu D. Exosomes in cancer development, metastasis, and immunity. Biochim Biophys Acta Rev Cancer. 2019;1871(2):455-468.

20. Zhang Y, Liu Y, Liu H, Tang WH. Exosomes: biogenesis, biologic function and clinical potential. Cell Biosci. 2019; 9:19.

21. Mollaei, H, Safaralizadeh R. Pouladi N. A brief review of exosomes and their roles in cáncer". Meta Gene. 2017;11: 70-74.

22. Kahlert C, Kalluri R. Exosomes in tumor microenvironment influence cancer progression and metastasis. $\mathrm{J}$ Mol Med (Berl). 2013;91(4):431-437.

23. Kalluri R, LeBleu VS. The biology, function, and biomedical applications of exosomes. Science. 2020;367(6478):eaau6977.

24. Colombo M, Raposo G, Théry C. Biogenesis, secretion, and intercellular interactions of exosomes and other extracellular vesicles. Annu Rev Cell Dev Biol. 2014; 30:255289. 
25. Sánchez OF, Rodríguez AV, Velasco-España JM, Murillo LC, Sutachan JJ, Albarracin SL. Role of Connexins 30, 36, and 43 in Brain Tumors, Neurodegenerative Diseases, and Neuroprotection. Cells. 2020;9(4):846.

26. Uzu M, Sin WC, Shimizu A, Sato H. Conflicting Roles of Connexin43 in Tumor Invasion and Growth in the Central Nervous System. Int J Mol Sci. 2018;19(4):1159.

27. Sin WC, Crespin S, Mesnil M. Opposing roles of connexin43 in glioma progression. Biochim Biophys Acta. 2012;1818(8):20582067.

28. Kameritsch P, Pogoda K, Pohl U. Channelindependent influence of connexin 43 on cell migration. Biochim Biophys Acta. 2012;1818(8), 1993-2001.

29. Beltrán A, Hernández-Aragón L, Galindo Ramírez F. Estudio del cambio en el nivel intracelular de $\mathrm{Ca}^{2+}$ y su propagación en células de una línea tumoral de pulmón generados por la activación de una sustancia fotosensible. 5a Reunión Anual del Colegio Mexicano para la Investigación del Cáncer. 2019; disponible en: https://www-optica.inaoep.mx/ tecnologia_salud/2019/trabajos_cartel.php
30. Castano AP, Demidova TN, Hamblin MR. Mechanisms in photodynamic therapy: part two-cellular signaling, cell metabolism and modes of cell death. Photodiagnosis Photodyn Ther. 2005;2(1), 1-23.

31. Whiteside TL. Tumor-Derived Exosomes and Their Role in Cancer Progression. Adv Clin Chem. 2016;74, 103-141.

32. Villagrasa A, Álvarez PJ, Osuna A, Garrido JM, Aránega A,Rodríguez-Serrano F. Exosomes Derived from Breast Cancer Cells, Small Trojan Horses? J Mammary Gland Biol Neoplasia. 2014;19(3-4): 303-313.

33. Yang L, Wu XH, Wang D, Luo CL, Chen LX. Bladder cancer cell-derived exosomes inhibit tumor cell apoptosis and induce cell proliferation in vitro. Mol Med Rep. 2013;8(4), 1272-1278. 